\title{
Diabetes in the elderly
}

\author{
Katarzyna Mordarska ${ }^{1}$, Małgorzata Godziejewska-Zawada² \\ ${ }^{1} 1^{\text {st }}$ Department of Internal Medicine, Bielański Hospital, Warsaw, Poland \\ ${ }^{2}$ Department of Endocrinology, Centre of Postgraduate Medical Education, Bielański Hospital, Warsaw, Poland
}

\begin{abstract}
The prevalence of both type 2 diabetes and prediabetes increases with advancing age. The most important factors leading to hyperglycaemia are as follows: deficiency of insulin secretion developing with age, and growing insulin resistance caused by a change in body composition and sarcopaenia.

Clinical features of diabetes in the elderly could be different. Diabetes in elderly people is often diagnosed with delay due to atypical symptoms (dementia, urinary incontinence) and occurrence of mainly postprandial hyperglycaemia. Elderly people are more exposed to diabetes complications, have more risk of myocardial infarction and end-stage renal disease, and are hospitalised more often due to hypoglycaemia than are younger patients.

Elderly people with diabetes are a heterogeneous group with different life expectancy, concomitant of chronic diseases, and the ability to self-control blood glucose or give themselves an injection. The therapy should be individualised. Older people with long-term diabetes and numerous chronic complications need a more liberal approach to reach specific goals of therapy. Additional goals should be avoiding hypoglycaemia, safety of the therapy, and its acceptance by the patient.
\end{abstract}

Key words: type 2 diabetes, elderly, insulin resistance, sarcopaenia, hypoglycaemia.

\section{Introduction}

Age is one of the most important risk factors in the development of prediabetes and type 2 diabetes [1]. A visible growth in of population of elderly people has been observed around the world for sedveral decades, and it is estimated that this trend will continue. According to GUS data from the year 2014, $15 \%$ of the population of Poland are older than 65 years; for comparison, in 1989 this age group accounted for only $10 \%$ of the population. During this period the number of people aged over 80 years living in Poland has doubled.

\section{Pathophysiology}

The process of aging of the human body leads to impairment of energy homeostasis and abnormalities in carbohydrate metabolism. The most important causes of hyperglycaemia are thought to be deficiency of insulin secretion developing with age and growing insulin resistance [2]. The Baltimore Longitudinal Study of Aging demonstrated that insulin secretion after glucose load decreases with age even after taking into account the influence of obesity and distribution of adipose tissue. Tests on the kinetics of insulin excretion in elderly people revealed that in comparison with younger people postprandial excretion of insulin is irregular and the amplitude of consecutive insulin pulses is lower [3]. Impairment of function of $\beta$ cells and dysfunction of insulin secretion in people suffering from type 2 diabetes are even deeper, and they are connected with almost total loss of the first phase of insulin secretion.

Moreover, the sensitivity of pancreatic $\beta$ cells for incretins decreases in the elderly. The effectiveness of incretins is smaller and it results in lower postprandial insulin levels and weaker suppression of glucagon secretion [1]. The next important factor leading to increased glucose level in blood is insulin resistance, which grows with age [4]. Distribution of adipose tissue in the elderly is changing (increased amount of visceral adipose tissue), and the amount of fat tissue grows in contrast to muscle mass, which decreases with age. The result of the process of aging is dysregulation of the hypothalamic-pituitary-adrenal axis (HPA axis), which leads to a relative prevalence of cortisol. Cortisol, as a catabolic hormone, is responsible for proteolysis, and its higher level leads to reduced muscle mass. Additionally, cortisol leads to hepatic insulin resistance. With age, muscles lose strength and mass (this phenomenon is called sarcopaenia). Insulin-dependent glucose uptake by skeletal muscles is reduced due to suppression of insulin receptors and glucose transporter GLUT-4 [5]. It has been demonstrated 
that in the seventh decade of life the mass of muscle tissue is reduced by $30-40 \%$ in favour of adipose tissue. Reduced physical activity of elderly people escalates this process. Co-existing obesity, increased adrenergic tension connected with age, decline in kidney functioning, and use of potentially diabetogenic drugs (diuretics, beta-adrenolytics, corticosteroids, psychotropic drugs, amiodarone) are additional factors promoting impairment of glucose metabolism and diabetes in the elderly.

Research on longevity carried out on a group of healthy centenarians revealed that glucose tolerance and insulin sensitivity in the oldest age group are definitely better than in persons aged 60-84 years [7]. Insulin resistance increases with age, but only until the age of about 85-90 years. Afterwards a significant improvement in tissue response to insulin occurs, comparable with people aged 20-30 years. An epidemiological study demonstrated that the frequency of occurrence of diabetes in centenarians is almost $50 \%$ lower than in people aged 65-84 years [6].

\section{Prevention}

Because the number of people with diabetes is still growing, preventative actions have been evaluated. In the Diabetes Prevention Program (DPP) it has been documented that interventions connected with changing life style (diet and physical activity) are most effective in the elderly with diabetes aged over 60 years. The risk of diabetes in the intervention group declined by $71 \%$ in comparison with the control group. Regular physical effort is beneficial to prevent sarcopaenia and obesity and improves insulin sensitivity. On the other hand, using metformin in elderly people did not prevented diabetes in comparison to younger people, in whom metformin decreased the risk of diabetes by $31 \%$ [8].

\section{Differences in the clinical picture of hyperglycaemia}

Modified physical activity and concomitant diseases can change the clinical picture of diabetes. In some elderly people the course of disease is asymptomatic. Osmotic diuresis in seniors could produce symptoms like nocturia, urinary incontinence, and urinary system infections. Additionally, elderly people have an impaired thirst mechanism, which increases the risk of dehydration. Dehydration leads to tiredness and deficiency of cognitive functions. Diabetes causes a change in rheological properties of blood resulting in excessive blood clotting. It increases the risk of a stroke, acute coronary syndromes, or intermittent claudication and worsens normal functioning for elderly people. These medical conditions can also be the first symptom of diabetes that was not recognised earlier.
Hyperglycaemia changes the hydration of optical structures of the eye, resulting in refraction impairment and worsening visual acuity, which makes physical activity more difficult and increases the risk of falling. Additionally, recurring infections, more difficult wound healing, and dementia, which are taken for granted in an elderly organism, could hide the course of diabetes [9].

\section{Prognosis}

The prognosis for seniors with diabetes is worse than for their peers without diabetes, and on average they live a few years less [10]. In the group of people with diabetes, patients aged over 75 years have the greatest risk of developing chronic complications, both microand macroangiopathic. These persons are more prone to myocardial infarction and developing end-stage renal disease, and are hospitalised twice as often due to hypoglycaemia [11]. The time of diagnosis of diabetes has some influence - the prognosis is better for patients who had diabetes diagnosed in old age. These people usually have a lower percentage of glycated haemoglobin and they require treatment with insulin less often in comparison with seniors suffering from long-term diabetes, who have the greatest risk of complications.

\section{Diagnosis}

According to the recommendations of Polish Diabetes Association for 2016, the criteria of recognising diabetes in elderly people remain the same as for younger age groups. It is generally known that postprandial hyperglycaemia starts to dominate with age, sometimes with correct fasting glucose level [12]. That is why the elderly with many risk factors for diabetes should not only control fasting glycaemia but also perform oral glucose tolerance test (OGTT with $75 \mathrm{~g}$ of glucose), which gives a chance to detect the disease early. Alternatively, it is possible to evaluate the glycated haemoglobin level. Although incorrect results in Poland are not enough to recognise diabetes $\left(\mathrm{HbA}_{1}>6.5 \%\right.$ according to ADA is enough to detect diabetes), it might be a recommendation to perform OGTT.

\section{Treatment}

The key rule of dealing with very heterogeneous group of elderly patients is an individual approach to therapy considering numerous factors. The most important ones are: life expectancy, engagement of the patient in the treatment process, the ability to do injections and perform blood glucose self-control, as well as occurrence of frailty and other co-existing diseases. Seniors with diabetes develop Alzheimer's disease [13] and depression [14] more often than persons without diabetes. The most important goal for the elderly is 
to improve or at least maintain their current quality of life. The treatment goal is to maintain as good glucose levels as possible without hypoglycaemia - sometimes it means higher glucose levels than in young age. The risk of hypoglycaemia increases with age and is greatest with insulin treatment; it is also relatively high with sulphonylureas. Each occurrence of hypoglycaemia increases the risk of death due to cardiological conditions and can cause worsening of cognitive functions or escalate dementia. Clinical manifestation of hypoglycaemia in elderly patients may occur with lower levels of glycaemia in comparison with younger persons [15]. Probably due to atypical symptoms, patients are often unaware of hypoglycaemia. Warning signals caused by stimulation of the adrenergic system, like sweating, tremor, or hunger may not occur at all. Additionally, counter-regulatory response of glucagon secretion is often limited and insufficient in senior patients. According to ACCORD research, intensive treatment of diabetes increases the risk of death in patients with a high cardiovascular risk. That is why in elderly patients with cardiovascular complications, more liberal goals of blood glucose levels have been established with target glycated haemoglobin $A_{1 c} \leq 8 \%[16]$.

Due to eating habits maintained for many years, dietary change is not very effective and should be recommended only in a range that is acceptable. An additional problem of elderly people is undernutrition, which according to PolSenior research is a common phenomenon in Poland, most visible in the eldest age group over 85 years old. Caloric deficiency leads to compensatory catabolism both in adipose and muscle tissues. It has been demonstrated that undernutrition and sarcopaenia correlate with death risk [17]. Decreased appetite, skipping meals, and caloric deficiency can make diabetes therapy, particularly insulin therapy, very difficult.

Physical effort, as recommended for seniors, must be adapted to individual abilities and physical fitness. It should be characterised by a gentle start and gentle finish, without flexing exercises.

Pharmacotherapy of diabetes in advanced age does not differ basically from pharmacotherapy for younger people with diabetes. During treatment, it is necessary to consider a higher number of side effects of medications and drug interactions due to polypragmasia. As far as possible, treatment should be simple - it is best to start with monotherapy and slowly increase medication doses.

\section{Metformin}

Metformin is still a first-choice drug, also for elderly people. Its safety in the treatment of seniors has been confirmed in several studies. Its advantages are: efficiency in decreasing $\mathrm{HbA} 1 \mathrm{c}$ level, beneficial effect on body mass and lipid profile, and the fact that metformin is a medication which improves the prognosis [18].
Nevertheless, this drug has its limitations when used with seniors. It is not recommended for persons who suffer from renal insufficiency (GFR $<30 \mathrm{ml} / \mathrm{min} / 1.73 \mathrm{~m}^{2}$ ), cardiac insufficiency (NYHA class III and IV), and chronic respiratory failure. Metformin should be also stopped in hypoxia (e.g. cardiac infarction, stroke, severe infection, dehydration). Therapy should start with small doses, e.g. $500 \mathrm{mg}$ once a day, which are consecutively increased every 7-14 days until the final dose is obtained, to avoid side effects causing problems with the digestive system. The maximum dose in elderly should be 2000 mg or less, depending on renal insufficiency.

\section{Sulphonylureas}

Sulphonylureas are another group of medicaments often used in diabetes treatment, also with seniors. These drugs are effective in decreasing glycaemia, are characterised by relatively good tolerance and are often attached to metformin or used in monotherapy if there is a contraindication to use biguanide. A serious side effect of sulfonylureas is a risk of hypoglycaemia, which is 2-3 times higher in comparison to other orally administered drugs. The risk of hypoglycaemia increases in cases of renal or hepatic insufficiency as well as with simultaneous use of drugs that increase the effect of sulfonylureas, such as: salicylates, acenocumarol, or fibrates. Gliclazide is safer than glimepiride [19] and the European Union Geriatric Medicine Society recommends the use of gliclazide with the elderly with diabetes and risk of hypoglycaemia. Contrary to glimepiride, gliclazide is metabolised to inactive metabolites, which leads to a lower risk of hypoglycaemia in case of worsening kidney function.

\section{Gliptins}

Gliptins - dipeptidyl peptidase-4 inhibitors (iDPP-4) - are a new group of medications successfully used in elderly people. DPP-4 inhibitors raise endogenous incretin levels (mainly glucagon-like peptide-1 - GLP-1), which increases insulin secretion and inhibits glucagon release. These drugs have a safe profile, and good efficiency and tolerance. It is suspected that they can have a protective effect on beta cells in the pancreas, which may delay the necessity of using insulin therapy. Another advantage of some drugs from this group is the possibility to use them in patients with renal insufficiency. These medications are not refundable in Poland, which limits their use on a large scale.

\section{Glucagon-like peptide-1 analogues}

Glucagon-like peptide-1 (GLP-1) analogues are another group of drugs whose market is systematically developing. These medications are effective in decreasing glycaemia, at the same time lowering the risk of hypo- 
glycaemia. Another advantage is their beneficial effect on the cardiovascular system (LEADER study with liraglutide). Nevertheless, the influence of these drugs on reducing body mass - which is a desirable effect in most patients with type 2 diabetes - in elderly people may increase sarcopaenia and risk of falling as well as undernutrition. Another drawback is the means of administration of this drug, as subcutaneous injections. The last problem is similar to the case of gliptins - lack of refund and high price.

\section{Thiazolidinediones}

The only drug from this group available in Poland is pioglitazone. Due to its effect on PPAR-gamma receptors, this drug influences transcription of numerous genes, leading to improvement of peripheral tissue sensitivity for insulin, mainly adipose tissue. It also has a beneficial effect on lipid profile. Unfortunately, this drug has a lot of limitations, particularly for the elderly. It causes fluid retention (it is contraindicated in the case of cardiac insufficiency), it can intensify and generate diabetic macular oedema, which is a severe ophthalmological complication that may lead to blindness, it decreases bone mineral density, and increases the risk of fractures. Thus, it is not the best therapeutic option for seniors.

\section{Flozins}

Flozins - inhibitors of type 2 renal sodium-glucose co-transporter (iSGLT - 2) - are the newest group of antidiabetic medications. Flozins inhibit re-uptake of glucose in renal proximal tubule, resulting in an increase in glucose excretion in urine. It leads to decreased glucose level in the blood and loss of calories. This therapy relates to low risk of hypoglycaemia, which is an important advantage. Moreover, the EMPA-REG study, which evaluates cardiovascular safety of empagliflozin, has demonstrated that this therapy is not only safe but also improves the prognosis and reduces the risk of cardiac death. Unfortunately, a slightly higher number of strokes during therapy has been observed. This should be checked in further observations. Additionally, treatment-induced glucosuria increases the risk of genitourinary system infections. It may be a problem for seniors, with whom the risk of infections is higher anyway. Urinary incontinence, worse hygiene due to disability, and diabetes itself could lead to bacterial and candida infections. Nevertheless, these medications may be a good therapeutic option for elderly people. This year we are expecting publication of the results of research on canagliflozin (CANVAS), and in 2019 on dapagliflozin (DECLARE) and ertugliflozin (VERTIS), which may consolidate the position of this group of drugs on the market of anti-diabetic medications.

\section{Insulin}

Insulin is still a necessity in the case of some elderly people when orally administered drugs have to be discontinued due to lack of efficacy or contraindications (e.g. because of renal insufficiency). According to PTD recommendations for 2016, insulin therapy should be introduced without delay if there are indications for it. The safety of this therapy largely depends on the possibility to co-operate with the patient. Diabetologists should evaluate a patient's cognitive functions as well as care and attention of the family. If possible, it is worth starting the treatment from preparations of the lowest risk of hypoglycaemia, which means from basal insulin (NPH or long-lasting analogue). Some elderly patients may need only short-acting insulin before meals. In the case of problems with appetite, rapid acting analogues may be given after a meal, after evaluating the amount of consumed food. Some people who lead a very regular life and whose demand for insulin is relatively low can be treated with insulin mixtures administered twice a day. For many people with type 2 diabetes this therapy is friendlier than four injections a day and easier for carers. In the case of insulin therapy for seniors, there are no uniform rules; the most important rule is taking an individual approach to the patient, considering their eating habits, mental skills, and physical fitness, as well as social conditions and the possibility of getting help from the family and friends.

\section{Hypoglycaemia in elderly patients}

Among adults of all ages, elderly people have the highest risk of hypoglycaemia as a complication of diabetes treatment [20]. Loss of body mass connected with age and frailty syndrome can result in a relevant decrease in the demand for anti-diabetic medications, both orally administered and insulin. Similarly, kidney function decreasing with age may cause the situation when the doses of drugs stimulating insulin secretion or insulin doses, which so far have been correctly controlling the course of the disease, become too high [21]. Then a significant change of treatment may be necessary. According to the recommendations of the European Union Geriatric Medicine Society, in the case of patients older than 60 years, and definitely older than 70 years, the treatment should be changed to gliclazide if the patient takes glimepiride. For patients who take insulin, doses should be gradually adjusted to prevent hypoglycaemia. For example, despite previous long-term treatment with four doses of insulin, frailty may cause a necessity to significantly reduce or even resign from the basal dose, leaving only pre-meal doses of insulin. A risk factor of severe hypoglycaemia is also recent hospital discharge [22]. Large doses of insulin or anti-diabetic drugs administered in hospital 
are not needed when glucotoxicity disappears. That is why each patient leaving hospital should be informed about the fact that demand for medications may be lower, and they should have the possibility to see a doctor in case of problems with decreasing doses of drugs and insulin. These problems show that a necessary factor in proper diabetes therapy, also in advanced age, is systematic blood glucose self-monitoring. A similar problem with drugs doses may arise with treatment of hypertension. Losing weight connected with frailty syndrome may significantly decrease demand for antihypertensive drugs and increase the risk of orthostatic hypotension and falling.

\section{Geriatric conditions connected with diabetes}

\section{Dementia and Alzheimer's diseose}

The Luchsinger study found a connection between diabetes and increased risk of dementia and Alzheimer's disease [23]. An impact of insulin resistance and hyperglycaemia on the development of dementia, both vascular and Alzheimer's type, has been described. Insulin may have some regulatory effect on tau protein and $\beta$-amyloid metabolism - important factors in pathogenesis of Alzheimer's disease. Hyperinsulinism may intensify inflammation and increase the $A \beta_{42}$ level in the brain, which can lead to an increased risk of Alzheimer's disease. On the other hand, recurring episodes of hypoglycaemia, which are a consequence of diabetes treatment, deteriorate cognitive functions and are connected with faster development of dementia [24].

\section{Frailty syndrome}

Frailty syndrome in geriatrics means that an organism lacks the ability to maintain the homeostasis and react to stress properly. It leads to loss of body mass, sarcopaenia, undernutrition, tiredness, lack of physical activity, and as a consequence to increased risk of falling, developing disability, dependence on other people, hospitalisation, and death.

It has been demonstrated that increase of glycated haemoglobin $\mathrm{HbA}_{1 \mathrm{c}}$ is connected with a risk of occurrence of frailty syndrome [25]. Coexistence of both conditions leads to more frequent occurrences of diabetic complications (chronic and acute), loss of independence, and worse prognosis. Diabetes is a disease that accelerates aging processes and leads to discrepancy between "biological age" and chronological age, which is why frailty syndrome occurs in patients suffering from diabetes on average a few years earlier than in seniors without diabetes [26]. It should be taken into account particularly in the context of proposed treatment and evaluating the risk of complications. Treat- ment of diabetes in patients suffering from frailty syndrome must be flexible and should consider the fact that with development of weakness and loss of body mass, demand for medicaments or insulin may noticeably decrease or become unnecessary.

\section{Conclusions}

Old age is connected with numerous health problems. To evaluate a patient with diabetes it is necessary to take into account not only their chronological age, but also their biological age, physical fitness, intellectual capacity, occurrence of other chronic diseases, as well as the patient's motivation and support from family and friends. The basis of treating an elderly person is trying to improve or at least maintain their current quality of life. Symptoms of hyperglycaemia should be reduced gradually avoiding hypoglycaemia at the same time. If life expectancy is at least 10 years, the target value of glycated haemoglobin $\mathrm{HbA}_{1 c} \leq 7 \%$ is the same as in younger age groups. In the case of patients with long-term diabetes complications, a more liberal approach and target value $\mathrm{HbA}_{1 \mathrm{c}} \leq 8 \%$ are recommended.

Seniors suffering from diabetes are more prone to develop chronic micro- and macrovascular complications as well as hypoglycaemia and frailty syndrome. When planning diagnosis and treatment in the elderly, not only satisfactory glycaemic control must be taken into consideration, but also an overall evaluation of the patient's state.

\section{Disclosure}

Authors report no conflict of interest.

\section{References}

1. Chang AM, Halter JBA. Aging and insulin secrection. J Physiol Endocrinol Metebol 2003; 284: E7-12.

2. Barbieri M, Rizzo MR, Manzella D, et al. Glucose regulation and oxidative stress in healthy centenarians. Exp Gerontol 2003; 38: 137-143.

3. Meneilly GS, Ryan AS, Veldhuis JD, et al. Increased disorderliness of basal insulin release, attenuated insulin secretory burst mass and reduced ultradian rhythmicity of insulin secretion in older individuals. J Clin Endocrinol Metab 1997; 82: 4088-4093.

4. Krentz AJ, Viljoen A, Sinclair A. Insulin resistance: a risk marker for disease and disability in older person. Diabet Med 2013; 30: 535-548.

5. Colman E, Katzel LI, Rogus E, et al. Weight loss reduces abdominal fat and improves insulin action in middle-aged and older men with impaired glucose tolerance. Metabolism 1995; 44: 1502-1508.

6. Crandall J, Schade D, Ma Y, et al. Diabetes Prevention Program Research group. The influence of age on the effects of lifestyle modification and metformin in prevention of diabetes. J Gerontol Abiol Sci Med Sci 2006; 61: 1075-1081.

7. Motta M, Benati E, Capri M, et al. Diabetes mellitus in extreme longevity. Exp Gerontol 2008; 43: 102-105.

8. Sieradzki J (ed.). Cukrzyca. Tom 2. Via Medica, Gdańsk 2016; 507-508.

9. Tancredi M, Rosengren A, Svensson AM, et al. Excess mortality among persons with type 2 diabetes. N Engl J Med 2015; 373: 1720-1732.

10. Miller ME, Bonds DE, Gerstein H, et al. The effects of baseline characteristics, glycaemia treatment approach, and glycated haemoglobin 
concentration on the risk of severe hypoglycaemia: post hoc epidemiological analysis of the ACCORD study. BMJ 2010; 340: b5444.

11. Szoke E, Shrayyef MZ, Messing S, et al. Effect of aging on glucose homeostasis: accelerated deterioration of beta-cell function in individuals with impaired glucose tolerance. Diabetes Care 2008; 31: 539-543.

12. Lu FP, Lin KP, Kuo HK. Diabetes and the risk of multi-system aging phenotypes: a systematic review and meta-analysis. PLoS One 2009; 4: e4144.

13. Nouwen A, Winkley K, Twisk J, et al. European Depression in Diabetes (EDID) Research Consortium. Type 2 diabetes mellitus as a risk factor for the onset of depression: a systematic review and meta-analysis. Diabetologia 2010; 53: 2480-2486.

14. Bremer JP, Jauch-Chara K, Hallschmid S, et al. Hypoglycemia unawareness in older compared with middle-aged patients with type 2 diabetes. Diabetes Care 2009; 32: 1513-1517.

15. Cypryk K, Czupryniak L, Czech A, et al. Zalecenia kliniczne dotyczące postępowania u chorych na cukrzycę 2016. Stanowisko Polskiego Towarzystwa Diabetologicznego. Diabetol Klin 2016; 5: A52.

16. Ensrud KE, Ewing SK, Cawthon PM, et al. Osteoporotic Fractures in Men Research Group. A comparison of Frailty Indexes for the Prediction of Falls, Disability, Fractures and Mortality in Older Men. J Am Geriatr Soc 2009; 57: 492-498.

17. Intensive blood-glucose control with sulphonylureas or insulin compared with conventional treatment and risk of complications in patients with type 2 diabetes (UKPDS 33). UK Prospective Diabetes Study (UKPDS) Group. Lancet 1998; 352: 837-853.

18. Schernthaner G, Grimdali A, Di Mario V, et al. GUIDE Study: double blind comparison of once-daily gliclazide MR and glimepiride in type 2 diabetic patients. Eur J Clin Invest 2004; 34: 535-542.

19. Pathak RD, Schroeder EB, Seaquist ER, et al. O'Connor for the SUPREMEDM Study Group. Severe hypoglycemia requiring medical intervention in a large cohort of adults with diabetes receiving care in U.S. Integrated health care delivery systems: 2005-2011. Diabetes Care 2016; 39: 363-370.

20. Seaquist ER, Anderson J, Childs B, et al. Hypoglycemia and diabetes: a report of workgroup of the American Diabetes Association and the Endocrine Society. J Clin Endocrinol Metab 2013: 98: 1845-1859.

21. Chelliah A, Burge MR. Hypoglycaemia in elderly patients with diabetes mellitus: causes and strategies for prevention. Drugs Aging 2004; 21 : 511-530.

22. Luchsinger JA, Tang MX, Stern Y, et al. Diabetes Mellitus and Risk of Alzheimer's Disease and Dementia with Stroke in a Multiethnic Cohort. Am J Epidemiol 2001; 154: 635-641.

23. Whitmer RA, Karter AJ, Yaffe K, et al. Hypoglycemic episodes and risk of dementia in older patients with type 2 diabetes mellitus. JAMA 2009; 301: 1565-1572.

24. Blaum CS, Xue QL, Tian J, et al. Is hyperglycemia associated with frailty status in older women? J Am Geriatr Soc 2009; 57: 840-847.

25. Hubbard RE, Andrew MK, Fallah $\mathrm{H}$, et al. Comparison of the prognostic importance of diagnosed diabetes, co-morbidity and frailty in older people. Diabet Med 2010; 27: 603-606. 